Cochrane produit des revues systématiques dédiées à diverses thématiques de la recherche médicale, créant ainsi une base fiable pour la médecine fondée sur les données probantes. Depuis 2016, tous les résidents suisses bénéficient d'un accès libre et gratuit à la Cochrane Library. Deuxième contributeur dans notre série de quatre articles, le Dr Bruno Kissling, médecin de famille, nous fait part de ses expériences avec la Cochrane Library.

Dr méd. Christoph Bosshard Vice-président de la FMH, Responsable du département Données, démographie et qualité (DDQ)

\title{
La Cochrane Library et le médecin de famille
}

\author{
Bruno Kissling \\ Dr méd., généraliste à la retraite
}

1 The European Definition of General Practice / Family Medicine 2002 / 2011 Wonca Europe www.woncaeurope.org/ gp-definitions

2 Family medicine ICPC research using electronic medical records - Institut für Hausarztmedizin. 3 Andreas Sönnichsen, prof., Zentrum für Public Health, Faculté de médecine de l'Université de Vienne, service de médecine générale et de famille, premier président du comité directeur du réseau allemand pour la médecine basée sur les faits (Deutsches Netzwerk Evidenzbasierte Medizin, DNEbM), éditeu d'IWIMED, essence of medicine, avec directives spécifiques à l'intention des médecins de famille et des patients, coéditeu d'EbM-Guidelines: Evidenzbasierte Medizin für Praxis \& Klinik.

\section{De nombreux médecins de famille n'ont pas l'habitude d'utiliser la Cochrane Library}

La petite enquête que j'ai menée auprès de mes collègues a révélé qu'ils en sont pour la plupart là où j'en suis moi-même: ils ont certes entendu parler de l'accès gratuit à cette offre, mais n'en ont que très rarement fait usage jusqu'ici. Ils utilisent plus souvent d'autres sources d'informations, mieux adaptées à leurs besoins.

\section{Les particularités du métier de médecin de famille ${ }^{1}$ et ses exigences spécifiques à l'égard des outils décisionnels}

Un médecin de famille voit quotidiennement un grand nombre de patients, dans le cadre de consultations relativement courtes. Un sondage interne réalisé par FIRE $^{2}$ a montré qu'en moyenne, un médecin de famille aborde 2,7 problématiques par consultation. Une forte proportion de nos patients présente une maladie chronique, une polymorbidité ainsi qu'une grande fragilité. Souvent, leurs symptômes sont indissociables du contexte psychosocial et d'autres tableaux cliniques concomitants. Pour un médecin désireux de prendre des décisions tenant compte de la personne et de son contexte, le ressenti individuel du patient est tout aussi important que la significativité statistique d'une différence entre deux options thérapeutiques. Prises toutes ensemble, ces circonstances augmentent l'incertitude touchant au symptôme ou au problème et compliquent d'autant la prise de décision du médecin et du patient. Cette complexité pose des exigences particulières aux aides scientifiques à la décision.

\section{La Cochrane Library et le médecin de famille}

La Cochrane Library est-elle l'outil adapté pour soutenir le médecin et le patient dans leur prise de décision commune, dans la situation particulière de la médecine de premier recours?

\section{Résumé}

La Cochrane Library devrait être connue des médecins du monde entier. Grâce à Internet, on accède à ses pages en quelques secondes à peine. Depuis 2016, tous les résidents suisses bénéficient d'un accès libre et gratuit à cette ressource, grâce à une initiative commune de l'Académie suisse des sciences médicales, de l'Office fédéral de la santé publique et des bibliothèques universitaires suisses. Dans cet article, je m'efforcerai de répondre aux questions suivantes: Les médecins de famille connaissent-ils la Cochrane Library, et I'utilisent-ils? Peuvent-ils trouver dans les revues Cochrane des réponses aux questions souvent complexes auxquelles ils se trouvent confrontés dans le cadre de leur pratique?

Répondant à mon sondage par e-mail, le Prof. Andreas Sönnichsen ${ }^{3}$, médecin généraliste et chercheur s'intéressant en particulier à la médecine fondée sur les données probantes, a remis l'appréciation suivante: «En tant qu'outil point of care, la Cochrane Library n'est pas adaptée pour la pratique quotidienne du médecin de famille. Même s'il en a une certaine habitude, le médecin ne peut guère y effectuer de recherches durant une consultation. L'outil est mieux adapté à la formation continue ou aux recherches en amont, en soirée ou pendant le week-end. Chaque revue Cochrane se focalise en principe sur une problématique spécifique. Or les questionnements typiques de la médecine de premier recours ne sont que rarement abordés dans les revues Cochrane. Non pas parce que l'on aurait les médecins de famille, mais parce que seul un petit nombre d'études ont été consacrées aux problématiques typiques de cette branche de la médecine.»

Les conclusions à en tirer? Pour que la Cochrane Library puisse lui servir d'aide à la décision au cabinet, le médecin de famille doit définir très précisément son champ d'investigation.

\section{Aide à la résolution de cas complexes}

Pour pouvoir rédiger cet article en connaissance de cause, je fais des essais de recherche dans la Cochrane 
Library. Petit à petit, je découvre la structure complexe de cette base de données électronique, et les possibilités qu'elle offre d'effectuer des recherches dans des milliers de revues et des dizaines de milliers d'essais cliniques ${ }^{4}$. J'essaie de trouver une solution pour aider ma patiente présentant une hyperthyroïdie avec teneur élevée en anticorps anti-récepteurs à la TSH, caractéristique de la maladie de Basedow. Etant donné que ma patiente présente en même temps un goitre nodulaire modérément gênant, nous aimerions savoir si une strumectomie constituerait une thérapie adaptée, susceptible de régler les deux problèmes en même temps.

Dans la fenêtre de recherche, sur la page d'accueil de la Cochrane Library, je saisis les mots-clefs «morbus Basedow» et restreins ma recherche en choisissant l'option «Title Abstract Keyword». Les résultats de la recherche apparaissent: une revue intitulée «Intraoperative neuromonitoring versus visual nerve identification for prevention of recurrent laryngeal nerve injury in adults undergoing thyroid surgery». Si je choisis l'option «All Text», j'obtiens quatre rapports d'essais (trials), dont notamment «Drug treatment of immune hyperthyroidism (Basedow disease). Patient selection, long-term follow-up and prevention of recurrence» et «Basedow's disease - hemithyroidectomy and contralateral neartotal strumectomy in comparison with bilateral neartotal strumectomy". Jusque-là, pas de réponse qui corresponde exactement à ma demande. En saisissant le mot-clef "goiter», et en sélectionnant l'option «All Text», j'obtiens 31 revues Cochrane. Trois d'entre elles répondent, de manière indirecte, à ma question: «Total or near-total thyroidectomy versus subtotal thyroidectomy for multinodular non-toxic goitre in adults», «Antithyroid drug regimen for treating Graves' hyperthyroidism", et enfin "Radioiodine therapy versus antithyroid medications for Graves' disease». Je poursuis mes recherches dans "Advanced Search", avec le mot-clef «Basedow disease» associé à «drug therapy» et «surgery», et, parmi
Bruno Kissling

Weststrasse 25

CH-3005 Berne

bruno.kissling[at]live.com
7 revues, je trouve en plus de celles déjà apparues précédemment une revue intitulée "Thyroid surgery for Graves' disease and Graves' ophthalmopathy». La rubrique "Clinical Answers» présente les résultats de trois de ces revues de manière condensée.

\section{Des résultats pertinents pour conseiller le patient}

$\mathrm{Au}$ final, quatre revues et les "Clinical Answers» contiennent des informations permettant de répondre à ma question. Après une étude approfondie, je retiens les points suivants, à aborder lors de l'entretien avec ma patiente: une thyroïdectomie totale serait préférable à une thyroïdectomie partielle, et pourrait entrer en ligne de compte pour traiter simultanément la maladie de Basedow et le goitre. Elle exigerait toutefois une thérapie de substitution à vie avec des hormones thyroïdiennes et comporterait un risque accru de perturbation à vie du métabolisme du calcium. Pas de différence entre les deux méthodes concernant les chances de résorption de l'opthalmopathie ou le risque de parèse du nerf récurrent. La maladie de Basedow est traitée par voie médicamenteuse (méthimazole) durant 12 à 18 mois, avec titrage attentif pour diminuer les effets secondaires. Une thérapie de substitution subséquente à l'aide d'hormones thyroïdiennes ne protège pas d'une récidive. Quant à la thérapie à l'iode radioactif, elle ne présente pas d'avantage par rapport au méthimazole (le propylthiouracile n'a pas été testé), mais augmente le risque d'ophthalmopathie. Dans mon optique de médecin de famille, je vais proposer à ma patiente la procédure suivante pour parvenir à une décision commune: l'état de la recherche médicale indique qu'il serait préférable et plus sûr de commencer par traiter la maladie de Basedow par voie médicamenteuse. Puis, dans un deuxième temps, une fois la maladie de Basedow maîtrisée, on pourrait traiter le goitre, modérément gênant, par voie chirurgicale et de préférence avec une thyroïdectomie partielle, globalement moins risquée malgré un risque de récidive du goitre légèrement plus élevé qu'avec une thyroïdectomie totale.

\section{Conclusion}

Il vaut la peine de découvrir les possibilités de la Cochrane Library, et de parcourir les pages de cette immense base de données. Cela prend beaucoup de temps au début, mais avec un peu d'habitude, on apprend à s'y repérer. C'est parfois un défi de trouver des réponses pertinentes aux questions complexes typiques de la médecine de premier recours, mais, comme l'a montré mon essai de recherche, c'est tout à fait possible avec une recherche clairement ciblée. 